\title{
Improved opacity expansion for medium-induced parton splitting
}

\author{
Yacine Mehtar-Tani ${ }^{a}$ and Konrad Tywoniuk ${ }^{b}$ \\ ${ }^{a}$ Physics Department, Brookhaven National Laboratory, \\ Upton, NY 11973, U.S.A. \\ ${ }^{b}$ Department of Physics and Technology, University of Bergen, \\ 5007 Bergen, Norway \\ E-mail: mehtartani@bnl.gov, konrad.tywoniuk@uib.no
}

\begin{abstract}
We present a new expansion scheme to compute the rate for parton splittings in dense and finite QCD media. In contrast to the standard opacity expansion, our expansion is performed around the harmonic oscillator whose characteristic frequency depends on the typical transverse momentum scale generated in the splitting. The first two orders account for the high frequency regime that is dominated by single hard scatterings together with the regime of multiple soft scatterings at low frequency. This provides the tools to go beyond the leading logarithmic approximation and compare to the full numerical solution to the corresponding Schrödinger equation across a wide range of gluon frequencies. We investigate the sensitivity of our results to varying the separation scale that defines the leading order. Finally, the application to Monte Carlo event generators is discussed.
\end{abstract}

KEYwords: Heavy Ion Phenomenology, Jets

ARXIV EPRINT: 1910.02032 


\section{Contents}

1 Introduction 1

2 Spectrum of medium-induced gluons 2

3 Expanding around the harmonic oscillator $\quad \mathbf{5}$

3.1 Leading order: the harmonic oscillator approximation 6

$\begin{array}{lll}3.2 & \text { Next-to-leading order correction to the harmonic oscillator } & 7\end{array}$

$\begin{array}{lll}4 & \text { Numerics } & 10\end{array}$

5 Conclusions $\quad 12$

\section{Introduction}

Measurements of significant modifications of hard probes observables, in particular jets, in heavy ion collisions as compared to proton-proton collisions at RHIC and LHC have firmly established the prominent role of final-state interactions in the dense nuclear medium created in heavy-ion collisions. For large systems, radiative processes are the main mechanism responsible for the observed quenching effects [1] (for recent reviews see [2, 3]). Therefore a precise description of these processes is essential for a quantitative understanding of the mechanisms driving in-medium jet modification and probing non-equilibrium dynamics of the quark-gluon plasma (QGP).

For energetic particles propagating close to the light-cone through a QCD medium, the problem reduces to describing the (non-relativistic) dynamics in the transverse plane perpendicular to the direction of propagation. A formalism for dealing with multiple scattering in a QCD medium was developed by Baier-Dokshitzer-Mueller-Peigné-Schiff $[4,5]$ and Zakharov [6, 7], usually referred to as the BDMPS-Z formalism. ${ }^{1}$ This resummation [9] can also be cast as an expansion in medium opacity [10-13]. ${ }^{2}$

The regime of strong interactions can be approximated by diffusive broadening of the transverse momentum, governed by the diffusion coefficient $\hat{q}$. In this regime, characterized by the formation time of the radiation $t_{\mathrm{f}}$ being larger than the mean free path $\ell_{\mathrm{mfp}}$, i.e. $\ell_{\text {mfp }} \ll t_{\mathrm{f}} \lesssim L$, interference effects between subsequent scattering centers have to be taking into account leading to the Landau-Pomeranchuk-Migdal (LPM) suppression. In this regime, the transport coefficient $\hat{q}$ is proportional to the Coulomb logarithm which must be regulated in the UV by the typical transverse momentum acquired through many soft scatterings, $q_{\text {med }}^{2}$, see e.g. [16]. This approximation ceases to be valid for relatively hard emissions for which the dominant effect comes from single scattering with the medium $[17,18]$.

\footnotetext{
${ }^{1}$ See [8] for an analogous approach within thermal field theory.

${ }^{2}$ For a similar effort within the so-called higher-twist formalism, see [14, 15].
} 
Apart from a full numerical solution of the propagator [19-22], it is currently unclear how to account for both regimes in a semi-analytic fashion.

The sensitivity of $\hat{q}$ to a high-energy cut-off scale comes from the underlying assumption that the medium in heavy-ion collisions consists of dressed quasi-particles whose interaction cross-section is given by a Coulomb-like power law. A first step towards the unification of the two limits described above was undertaken in [23]. The main idea, inspired by the Moliére theory of scattering [24] (see also [25] for a more recent application in the context of momentum broadening in high energy proton-nucleus collisions), is to treat the leading logarithm generated by the Coulomb tail in the medium potential to all orders in opacity since in this case the problem simplifies to solving for a harmonic oscillator potential and the remainder is treated a perturbation. The expansion parameter will thus be $\ln ^{-1}\left(q_{\text {med }}^{2} / \mu^{2}\right)$, where $q_{\text {med }}$ is the typical transverse momentum acquired through multiple soft scatterings with the medium and $\mu$ an IR cut-off such as the Debye mass.

In this work, we generalize the approach in [23] to go beyond the leading-logarithmic corrections to $\hat{q}$. This is achieved by expanding the scattering kernel around a harmonic oscillator approximation to incorporate the effects of hard interactions in addition to multiple soft interactions. This is equivalent to a shift of the conventional opacity expansion around vacuum propagation to a solution that directly accounts for multiple soft scattering in the medium. Since the procedure in principle encodes the full information about the power-law behavior of Coulomb interactions, we also introduce an interpolation to the Bethe-Heitler regime of very soft gluon emissions, i.e. $t_{\mathrm{f}} \lesssim L$, which is especially relevant for dilute media, $\ell_{\mathrm{mfp}} \lesssim L$. The description of this regime is achieved through a prescription of the matching scale that removes the possibility for multiple scattering, see also [26] for a discussion of the matching scale. Our result for the spectrum of medium-induced splittings is therefore valid for arbitrary energies and medium sizes, interpolating between three regimes: 1) Bethe-Heitler $\left.\left(t_{\mathrm{f}}<\ell_{\mathrm{mfp}}\right), 2\right)$ LPM $\left(\ell_{\mathrm{mfp}}<t_{\mathrm{f}}<L\right)$ and 3) single, hard scattering $\left(L<t_{\mathrm{f}}\right)$. Although we implicitly assume that the jet is energetic enough such that $\sqrt{E / \hat{q}}>L$ (the "thin" medium limit, according to [18]), our improved formula also accounts for "thick" media where $L>\sqrt{E / \hat{q}}$.

The manuscript is organized as follows. In section 2, we recall the general expression for leading order splitting distribution in the presence of a dense QCD medium. In section 3, we evaluate the spectrum by expanding close to the harmonic oscillator. We carry out the analytic calculations for the first two terms that are sufficient to interpolate between multiple soft and single hard approximations. In section 4, our results for the spectrum and the rate are plotted. The latter is compared to the full numerical results from the McGill group which was first presented in [19].

\section{Spectrum of medium-induced gluons}

The probability for a high energy parton $a$, of energy $E$, to split into a two partons $b$ and $c$ carrying a fraction $z$ and $1-z$ of its energy, respectively, due to multiple scatterings in 
a dense QCD medium is given by

$$
z \frac{\mathrm{d} I_{b a}}{\mathrm{~d} z}=\frac{\alpha_{s} z P_{b a}(z)}{(z(1-z) E)^{2}} 2 \operatorname{Re} \int_{0}^{\infty} \mathrm{d} t_{2} \int_{0}^{t_{2}} \mathrm{~d} t_{1} \boldsymbol{\partial}_{\boldsymbol{x}} \cdot \boldsymbol{\partial}_{\boldsymbol{y}}\left[\mathcal{K}_{b a}\left(\boldsymbol{x}, t_{2} ; \boldsymbol{y}, t_{1}\right)-\mathcal{K}_{0}\left(\boldsymbol{x}, t_{2} ; \boldsymbol{y}, t_{1}\right)\right]_{\boldsymbol{x}=\boldsymbol{y}=0} .
$$

where $P_{b(c) \leftarrow a}(z)$ stands for the Altarelli-Parisi splitting functions that read for the various relevant branching processes

$$
\begin{aligned}
P_{\mathrm{gg}}(z) & =\frac{1}{2} 2 C_{A} \frac{[1-z(1-z)]^{2}}{z(1-z)} \\
P_{\mathrm{qg}}(z) & =\frac{1}{2} 2 N_{f} T_{F}\left(z^{2}+(1-z)^{2}\right), \\
P_{\mathrm{gq}}(z) & =\frac{1}{2} C_{F} \frac{1+(1-z)^{2}}{z}, \\
P_{\mathrm{qq}}(z) & =\frac{1}{2} C_{F} \frac{1+z^{2}}{(1-z)} .
\end{aligned}
$$

where $C_{A}=N_{c}, C_{F}=\left(N_{c}^{2}-1\right) / 2 N_{c}$ and $T_{F}=1 / 2$, with $N_{c}=3$ for $\mathrm{SU}(3) . N_{f}$ is the active number of quark flavors which we fix to 3 for our applications throughout. Note that we have adopted the instant form notations for simplicity. The time variables stand in fact for light-cone time, i.e., $t=x^{+}$and the the mass (energy) $E$ corresponds the the longitudinal light-cone momentum $p^{+}$.

The Green's function $\mathcal{K}\left(\boldsymbol{x}, t_{2} ; \boldsymbol{y}, t_{1}\right)$ accounts for the interactions with the medium taking place during the formation time of the splitting. It implicitly depends on the color representation of the in-coming and out-going partons and obeys the following Schödinger equation,

$$
\left[i \frac{\partial}{\partial t}+\frac{\partial^{2}}{2 z(1-z) E}+i v_{b a}(\boldsymbol{x}, t)\right] \mathcal{K}_{b a}\left(\boldsymbol{x}, t ; \boldsymbol{y}, t_{0}\right)=i \delta\left(t-t_{0}\right) \delta(\boldsymbol{x}-\boldsymbol{y})
$$

where medium interactions are incorporated via the interaction Hamiltonian $H_{\text {int }}=i v(\boldsymbol{x}, t)$. The free part of the non-relativistic Hamiltonian in $2+1$ dimensions is given by $H_{0}=$ $i \partial_{t}+\partial^{2} /(2 \omega)$ with a "mass" parameter given by $\omega \equiv z(1-z) E$ is solved by the following vacuum Green's function,

$$
\mathcal{K}_{0}\left(\boldsymbol{x}, t ; \boldsymbol{y}, t_{0}\right)=\frac{\omega}{2 \pi i\left(t-t_{0}\right)} \exp \left[i \frac{\omega(\boldsymbol{x}-\boldsymbol{y})^{2}}{2\left(t-t_{0}\right)}\right] .
$$

Naturally, for $z<1$ we can identify $\omega$ with the energy of the emitted, soft daughter particle, $\omega \approx z E$. Thus, eq. (2.6) describes the propagation through, and subsequent transverse broadening in a medium described by an imaginary three-body potential $i v_{b a}(\boldsymbol{x}, t)$, which is given by

$$
\begin{aligned}
v_{b a}^{c}(\boldsymbol{x}, t)= & \frac{C_{b}+C_{c}-C_{a}}{2} \tilde{v}(\boldsymbol{x}, t)+\frac{C_{c}+C_{a}-C_{b}}{2} \tilde{v}(z \boldsymbol{x}, t) \\
& +\frac{C_{a}+C_{b}-C_{c}}{2} \tilde{v}((1-z) \boldsymbol{x}, t),
\end{aligned}
$$


where $C_{a}, C_{a}$ and $C_{c}$ are the color factors associated with partons in representations $a, b$ and $c$, respectively. Explicitly, we have

$$
\begin{aligned}
& v_{\mathrm{gg}}(\boldsymbol{x}, t)=\frac{N_{c}}{2} \tilde{v}(\boldsymbol{x}, t)+\frac{N_{c}}{2} \tilde{v}(z \boldsymbol{x}, t)+\frac{N_{c}}{2} \tilde{v}((1-z) \boldsymbol{x}, t), \\
& v_{\mathrm{gq}}(\boldsymbol{x}, t)=\frac{N_{c}}{2} \tilde{v}(\boldsymbol{x}, t)+\left(C_{F}-\frac{N_{c}}{2}\right) \tilde{v}(z \boldsymbol{x}, t)+\frac{N_{c}}{2} \tilde{v}((1-z) \boldsymbol{x}, t), \\
& v_{\mathrm{qq}}(\boldsymbol{x}, t)=\frac{N_{c}}{2} \tilde{v}(\boldsymbol{x}, t)+\frac{N_{c}}{2} \tilde{v}(z \boldsymbol{x}, t)+\left(C_{F}-\frac{N_{c}}{2}\right) \tilde{v}((1-z) \boldsymbol{x}, t), \\
& v_{\mathrm{qg}}(\boldsymbol{x}, t)=\left(C_{F}-\frac{N_{c}}{2}\right) \tilde{v}(\boldsymbol{x}, t)+\frac{N_{c}}{2} \tilde{v}(z \boldsymbol{x}, t)+\frac{N_{c}}{2} \tilde{v}((1-z) \boldsymbol{x}, t) .
\end{aligned}
$$

where ${ }^{3}$ the potential is given by

$$
\tilde{v}(\boldsymbol{x}, t)=\int_{\boldsymbol{q}} \frac{\mathrm{d} \sigma_{\mathrm{el}}}{\mathrm{d}^{2} \boldsymbol{q}}\left(1-\mathrm{e}^{i \boldsymbol{q} \cdot \boldsymbol{x}}\right) .
$$

The elastic scattering potential with the medium can be extracted from thermal field theory in a weakly-coupled medium [27-29], but is often modeled as

$$
\frac{\mathrm{d}^{2} \sigma_{\mathrm{el}}}{\mathrm{d}^{2} \boldsymbol{q}} \equiv \frac{g^{4} n}{\left(\boldsymbol{q}^{2}+\mu^{2}\right)^{2}}
$$

also referred to the Gyulassy-Wang model [30] (here, $n=n(t) \sim T^{3}$ corresponds to the density of scattering centers in the medium). The potential is screened at the scale $\mu$ that is related to the Debye mass in a thermal medium, i.e., $\mu^{2} \sim m_{D}^{2}=\left(1+N_{f} / 6\right) g^{2} T^{2}$.

In the case of interest many scattering centers contribute during the branching process and, as a result, the typical transverse momentum acquired by the three body system $(a, b, c)$ is much larger than the Debye mass $k_{\perp} \sim x_{\perp}^{-1} \gg \mu$. As a result, $\tilde{v}(\boldsymbol{x}, t)$ is dominated by a large Coulomb logarithm $\ln \left(x_{\perp} \mu\right)^{-1}$. Using the GW model for the potential given by eq. (2.14), we find

$$
\begin{aligned}
\tilde{v}(\boldsymbol{x}, t) & =\int_{\boldsymbol{q}} \frac{\mathrm{d} \sigma_{\mathrm{el}}}{\mathrm{d}^{2} \boldsymbol{q}}\left(1-\mathrm{e}^{i \boldsymbol{q} \cdot \boldsymbol{x}}\right)=\frac{\hat{q}_{0}(t)}{\mu^{2} N_{c}}\left[1-\mu|\boldsymbol{x}| K_{1}(\mu|\boldsymbol{x}|)\right], \\
& \approx \frac{1}{4 N_{c}} \boldsymbol{x}^{2} \hat{q}_{0}(t)\left(\ln \frac{4}{\mu^{2} \boldsymbol{x}^{2}}+1-2 \gamma_{E}\right)+\ldots
\end{aligned}
$$

up to sub-leading corrections of order $\sim \boldsymbol{x}^{4}$. Here, $K_{1}(x)$ is the modified Bessel function of the second kind and $\gamma_{E} \approx 0.577 \ldots$ the Euler-Mascheroni constant. For later convenience we have introduced the "bare" quenching parameter, stripped of the Coulomb logarithm

$$
\hat{q}_{0}(t) \equiv 4 \pi \alpha_{s}^{2} N_{c} n(t) .
$$

For a thermal medium we simply have $n=(3 / 2) T^{3}$. For $T=0.4 \mathrm{GeV}$ and $g=1.94$ (so that $\left.\alpha_{s} \approx 0.3\right)$ we find $\hat{q}_{0}(t)=1.83 \mathrm{GeV}^{2} / \mathrm{fm}$ and $m_{D}=0.9 \mathrm{GeV}$. For the remaining part of the paper, we identify $\mu=m_{D}$, see also [26] for a more precise matching.

\footnotetext{
${ }^{3}$ Throughout, we adopt the shorthand $\int_{\boldsymbol{q}} \equiv \int \mathrm{d}^{2} \boldsymbol{q} /(2 \pi)^{2}$ and $\int_{\boldsymbol{x}} \equiv \int \mathrm{d}^{2} \boldsymbol{x}$ for the transverse integrals.
} 


\section{Expanding around the harmonic oscillator}

It is in generally difficult to solve eq. (2.6) exactly other than by using numerical methods $[20,21,29]$. A first strategy consists of a plain expansion in $v(\boldsymbol{x}, t)$, which corresponds to the standard opacity expansion, where the opacity is defined as a ratio between the mean free path and the medium length, $\sim \ell_{\mathrm{mfp}} / L$. The first order in the latter approach is typically referred to as the Gyulassy-Levai-Vitev (GLV) approximation [10], see also [9]. However, this approximation breaks down in a dense medium at frequencies $\omega<\omega_{c} \sim \hat{q} L^{2}$ which, for realistic values such as $\hat{q}=2 \mathrm{GeV}^{2} / \mathrm{m}$ and $L=5 \mathrm{fm}$, for instance, yields a large value $\omega_{c}=250 \mathrm{GeV}$. In this case, the transverse momentum accumulated during the branching is determined by multiple soft scattering, i.e. $k_{\perp}^{2} \sim \sqrt{\hat{q} \omega}$. In this nonperturbative regime the potential can be approximated by $v(\boldsymbol{x}, t) \sim \boldsymbol{x}^{2}$ by neglecting the variation of the Coulomb logarithm (cf. eq. (2.15)). In this case, the equation of motion is identical to that of a harmonic oscillator with complex frequency, hence this scheme is often referred to as the "harmonic oscillator" (HO) approximation. Of course, in order to obtain a quantitively sound result one needs to estimate the argument of the logarithm which introduces an uncertainty of order the inverse of the Coulomb logarithm.

Our strategy in what follows is to shift the expansion point to the "harmonic oscillator" solution as follows

$$
v(\boldsymbol{x}, t)=v_{\mathrm{HO}}(\boldsymbol{x}, t)+\delta v(\boldsymbol{x}, t),
$$

where $\delta v(\boldsymbol{x}, t)=v(\boldsymbol{x}, t)-v_{\mathrm{HO}}(\boldsymbol{x}, t) \ll v_{\mathrm{HO}}(\boldsymbol{x}, t)$, will be treated as a perturbation. The HO potential is given by

$$
v_{\mathrm{HO}}(\boldsymbol{x}, t) \equiv \frac{1}{4} \boldsymbol{x}^{2} \hat{q}_{\mathrm{eff}}(t),
$$

with the effective $\hat{q}$ parameter chosen to be

$$
\hat{q}_{\mathrm{eff}}\left(Q_{\mathrm{sub}}^{2}, t\right)=\frac{1}{2}\left[1+\left(\frac{2 C_{R}}{N_{c}}-1\right) z^{2}+(1-z)^{2}\right] \hat{q}_{0}(t) \ln \frac{a Q_{\mathrm{sub}}^{2}}{\mu^{2}} .
$$

where $\hat{q}_{0}(t)$ is given in eq. $(2.17)$ and $a=4 \mathrm{e}^{-2 \gamma_{E}+1}$. Here, we have limited our discussion to a parton in representation $R=q, g$ that radiates a gluon of energy fraction $z$. The constant $a$ accounts for the constant terms in eq. (2.15).

The jet quenching coefficient $\hat{q}_{\text {eff }}$ is logarithmically dependent on a subtraction scale which is only a function of $\omega$, i.e. $Q_{\text {sub }}=Q_{\text {sub }}(\omega)$. This scale must be chosen to be the typical transverse momentum generated during the splitting, that is,

$$
Q_{\mathrm{sub}}^{2} \simeq \sqrt{z(1-z) E \hat{\mathrm{q}}_{\mathrm{eff}}\left(Q_{\mathrm{sub}}^{2}\right)}
$$

in the HO approximation.

The advantage of choosing this expansion point, is that in the HO approximation the Schödinger equation,

$$
\left[i \frac{\partial}{\partial t}+\frac{\boldsymbol{\partial}^{2}}{2 \omega}+i v_{\mathrm{HO}}(\boldsymbol{x}, t)\right] \mathcal{K}_{\mathrm{HO}}\left(\boldsymbol{x}, t ; \boldsymbol{y}, t_{0}\right)=i \delta\left(t-t_{0}\right) \delta(\boldsymbol{x}-\boldsymbol{y})
$$


admits the known analytic solution [31],

$$
\mathcal{K}_{\mathrm{HO}}\left(\boldsymbol{x}, t ; \boldsymbol{y}, t_{0}\right)=\frac{i \omega}{2 \pi S\left(t, t_{0}\right)} \exp \left\{i \frac{\omega}{2 S\left(t, t_{0}\right)}\left[C\left(t_{0}, t\right) \boldsymbol{x}^{2}+C\left(t, t_{0}\right) \boldsymbol{y}^{2}-2 \boldsymbol{x} \cdot \boldsymbol{y}\right]\right\} .
$$

Here, the functions $S\left(t, t_{0}\right)$ and $C\left(t, t_{0}\right)$ represent the two independent solution of the equation

$$
\frac{\mathrm{d}^{2} f(t)}{\mathrm{d} t^{2}}=-\Omega^{2}(t) f(t)
$$

where the frequency is given by $\Omega(t)=\sqrt{\hat{q}(t) /(2 i \omega)}$, with boundary conditions $S\left(t_{0}, t_{0}\right)=0$ and $\left.\partial_{t} S\left(t, t_{0}\right)\right|_{t=t_{0}}=1$, and $C\left(t_{0}, t_{0}\right)=1$ and $\left.\partial_{t} C\left(t, t_{0}\right)\right|_{t=t_{0}}=0$, respectively. They are related through a constant Wronskian,

$$
W=C\left(t, t_{0}\right) \partial_{t} S\left(t, t_{0}\right)-S\left(t, t_{0}\right) \partial_{t} C\left(t, t_{0}\right) .
$$

Given the boundary conditions on the functions $S$ and $C$, it turns out that $W=1$. For a medium of constant density and length $L$, where $\Omega(t)=\Omega$, we find that $S\left(t, t_{0}\right)=$ $\sin \left[\Omega\left(t-t_{0}\right)\right] / \Omega$ and $C\left(t, t_{0}\right)=\cos \left[\Omega\left(t-t_{0}\right)\right]$.

The full solution can then be recast as an implicit equation, and reads

$$
\mathcal{K}\left(\boldsymbol{x}, t_{1} ; \boldsymbol{y}, t_{0}\right)=\mathcal{K}_{\mathrm{HO}}\left(\boldsymbol{x}, t_{1} ; \boldsymbol{y}, t_{0}\right)-\int \mathrm{d}^{2} \boldsymbol{u} \int_{t_{0}}^{t_{1}} \mathrm{~d} t \mathcal{K}_{\mathrm{HO}}\left(\boldsymbol{x}, t_{1} ; \boldsymbol{u}, t\right) \delta v(\boldsymbol{u}, t) \mathcal{K}\left(\boldsymbol{u}, t ; \boldsymbol{y}, t_{0}\right) .
$$

In what follows we shall solve the latter iteratively for the first two orders: the leading-order (LO) term reads $\mathcal{K}_{\mathrm{LO}}=\mathcal{K}^{(0)}$, where

$$
\mathcal{K}^{(0)}\left(\boldsymbol{x}, t_{1} ; \boldsymbol{y}, t_{0}\right)=\mathcal{K}_{\mathrm{HO}}\left(\boldsymbol{x}, t_{1} ; \boldsymbol{y}, t_{0}\right) .
$$

The next-to-leading (NLO) correction is given by

$$
\mathcal{K}^{(1)}\left(\boldsymbol{x}, t_{1} ; \boldsymbol{y}, t_{0}\right)=-\int \mathrm{d}^{2} \boldsymbol{u} \int_{t_{0}}^{t_{1}} \mathrm{~d} t \mathcal{K}_{\mathrm{HO}}\left(\boldsymbol{x}, t_{1} ; \boldsymbol{u}, t\right) \delta v(\boldsymbol{u}, t) \mathcal{K}_{\mathrm{HO}}\left(\boldsymbol{u}, t ; \boldsymbol{y}, t_{0}\right),
$$

so that the full NLO term is simply $\mathcal{K}_{\mathrm{NLO}}=\mathcal{K}^{(0)}+\mathcal{K}^{(1)}$. Higher orders are found in a analogous manner. Our results below show that the two first terms already provide a reasonable description of the spectrum and the related splitting rate.

\subsection{Leading order: the harmonic oscillator approximation}

Let us first consider the leading order that corresponds the BDMPS approximation. Inserting eq. (3.6) in eq. (2.1) yields

$$
z \frac{\mathrm{d} I^{(0)}}{\mathrm{d} z} \equiv z \frac{\mathrm{d} I_{\mathrm{HO}}}{\mathrm{d} z}=\frac{\alpha_{s} z P(z)}{\pi} 2 \operatorname{Re} \int_{0}^{\infty} \mathrm{d} t_{2} \int_{0}^{t_{2}} \mathrm{~d} t_{1}\left[\frac{1}{S^{2}\left(t_{2}, t_{1}\right)}-\frac{1}{\left(t_{2}-t_{1}\right)^{2}}\right]
$$

where we have left the indices indicating the parton splitting to be implicit. Using the following property [32]

$$
\partial_{t}\left(\frac{C(t, s)}{S(t, s)}\right)=-\frac{1}{S^{2}(t, s)}
$$


the $t_{2}$ integration can be carried out and reads

$$
\int_{t_{1}}^{\infty} \mathrm{d} t_{2} \frac{1}{S^{2}\left(t_{2}, t_{1}\right)}=\frac{C\left(t_{1}, t_{1}\right)}{S\left(t_{1}, t_{1}\right)}-\frac{C\left(\infty, t_{1}\right)}{S\left(\infty, t_{1}\right)} .
$$

The first term in eq. (3.14) cancels against the vacuum piece, i.e. the second term in eq. (3.12), while the second one can be integrated further over $t_{1}$,

$$
\int_{0}^{\infty} \mathrm{d} t_{1} \frac{C\left(\infty, t_{1}\right)}{S\left(\infty, t_{1}\right)}=-\int_{0}^{\infty} \mathrm{d} t_{1} \frac{\partial_{t_{1}} C\left(t_{1}, L\right)}{C\left(t_{1}, L\right)}=\ln C(0, L)=\ln \cos (\Omega L),
$$

where we have used the decomposition of $C(\infty, s)$ and $S(\infty, s)$ as a superposition of other solution to the wave equation [32],

$$
\begin{aligned}
& S\left(t, t_{1}\right)=C\left(t_{1}, t_{0}\right) S\left(t, t_{0}\right)-S\left(t_{1}, t_{0}\right) C\left(t, t_{0}\right), \\
& C\left(t, t_{1}\right)=-\partial_{t_{1}} C\left(t_{1}, t_{0}\right) S\left(t, t_{0}\right)-\partial_{t_{1}} S\left(t_{1}, t_{0}\right) C\left(t, t_{0}\right) .
\end{aligned}
$$

Hence, letting $t=\infty, t_{1}=s$ and $t_{0}=L$ yields

$$
\begin{aligned}
& S(\infty, s)=C(s, L) S(\infty, L)-S(s, L) C(\infty, L), \\
& C(\infty, s)=-\partial_{s} C(s, L) S(\infty, L)+\partial_{s} S(s, L) C(\infty, L) .
\end{aligned}
$$

Finally, inserting eq. (3.15) into eq. (3.12) yields the BDMPS-Z result

$$
z \frac{\mathrm{d} I^{(0)}}{\mathrm{d} z}=\frac{2 \alpha_{s}}{\pi} z P(z) \ln |\cos (\Omega L)| .
$$

Eq. (3.18) encompasses two regimes

$$
z \frac{\mathrm{d} I^{(0)}}{\mathrm{d} z} \simeq \frac{\alpha_{s}}{\pi} z P(z) \begin{cases}\sqrt{\frac{\omega_{c}}{2 \omega}} & \text { for } \quad \omega \ll \omega_{c} \\ \frac{1}{12}\left(\frac{\omega_{c}}{\omega}\right)^{2} & \text { for } \quad \omega \gg \omega_{c}\end{cases}
$$

expressed in terms of the characteristic frequency

$$
\omega_{c}=\frac{1}{2} \hat{q}_{\mathrm{eff}} L^{2}
$$

Recall that $q_{\mathrm{eff}}$ is a function of $z$ and depends on the flavor of the partonic configuration (cf. eq. (3.3)).

\subsection{Next-to-leading order correction to the harmonic oscillator}

Let us turn now to evaluating the next-to-leading term, given as a sum of eqs. (3.10) and (3.11). The physical meaning of the NLO correction is that one "soft" scattering, described purely via diffusive transverse momentum broadening, is replaced by a "hard" scattering, i.e. an interaction with the medium described by the Coulomb potential. This 
opens for the possibility that a single, hard kick from the medium can dominate the total transverse momentum transversed from the medium during the formation time. The correction to the splitting distribution reads, see [23] for more details,

$$
z \frac{\mathrm{d} I^{(1)}}{\mathrm{d} z}=\frac{\alpha_{s} z P(z)}{\pi^{2}} 2 \operatorname{Re} \int_{0}^{L} \mathrm{~d} s \int \frac{\mathrm{d}^{2} \boldsymbol{u}}{\boldsymbol{u}^{2}} \delta v(\boldsymbol{u}, s) \mathrm{e}^{-k^{2}(s) \boldsymbol{u}^{2}},
$$

where the indices are suppressed and

$$
k^{2}(s)=i \frac{\omega}{2}\left[\frac{C(0, \infty)}{S(0, \infty)}-\frac{C(\infty, s)}{S(\infty, s)}\right] .
$$

In particular, for a medium with constant density $n(s)=n \Theta(L-s)$, we find

$$
k^{2}(s)=i \frac{\omega \Omega}{2}[\cot (\Omega s)-\tan (\Omega(L-s))] .
$$

For simplicity and without loss of generality, let us focus on the case where a gluon of energy fraction $z$ is emmitted of a parton $R$. Then,

$$
\delta v_{\mathrm{gR}}(\boldsymbol{x}, t)=\frac{N_{c}}{2} \delta \tilde{v}(\boldsymbol{x}, t)+\left(C_{R}-\frac{N_{c}}{2}\right) \delta \tilde{v}(z \boldsymbol{x}, t)+\frac{N_{c}}{2} \delta \tilde{v}((1-z) \boldsymbol{x}, t)
$$

where

$$
\delta \tilde{v}(\boldsymbol{x}, t)=\int_{\boldsymbol{q}} \frac{\mathrm{d}^{2} \sigma_{\mathrm{el}}}{\mathrm{d} \boldsymbol{q}^{2}}\left(1-\mathrm{e}^{i \boldsymbol{q} \cdot \boldsymbol{u}}\right)-\frac{1}{4 N_{c}} \boldsymbol{u}^{2} \hat{q}_{0} \ln \frac{Q_{\mathrm{sub}}^{2}}{\mu^{2}}
$$

In order to integrate over $\boldsymbol{u}$ it is convenient to use the Fourier representation of the dipole cross-section. Consider for instance the contribution from the second term in eq. (3.24),

$$
\begin{aligned}
& \left.z \frac{\mathrm{d} I^{(1)}}{\mathrm{d} z}\right|_{a}=\frac{\alpha_{s} z P(z)}{\pi^{2}}\left(C_{R}-\frac{N_{c}}{2}\right) 2 \operatorname{Re} \int_{0}^{L} \mathrm{~d} s \int \frac{\mathrm{d}^{2} \boldsymbol{u}}{\boldsymbol{u}^{2}} \int_{\boldsymbol{q}} \frac{\mathrm{d}^{2} \sigma_{\mathrm{el}}}{\mathrm{d} \boldsymbol{q}^{2}}\left(1-\mathrm{e}^{i z \boldsymbol{q} \cdot \boldsymbol{u}}\right) \mathrm{e}^{-k^{2}(s) \boldsymbol{u}^{2}}, \\
& \left.z \frac{\mathrm{d} I^{(1)}}{\mathrm{d} z}\right|_{b}=-\frac{\alpha_{s} z P(z)}{\pi^{2}}\left(C_{R}-\frac{N_{c}}{2}\right) 2 \operatorname{Re} \int_{0}^{L} \mathrm{~d} s \int \mathrm{d}^{2} \boldsymbol{u} \frac{1}{4} z^{2} \hat{q}_{0} \mathrm{e}^{-k^{2}(s) \boldsymbol{u}^{2}} .
\end{aligned}
$$

The contribution $a$ relates to the compete dipole cross-section from which the HO part, i.e. contribution $b$, must be subtracted. Strikingly, the integrations can be performed analytically by noticing that the $u$ integral yields

$$
\int_{0}^{\infty} \frac{\mathrm{d} u}{u}\left[1-J_{0}(z q u)\right] \mathrm{e}^{-u^{2} k^{2}(s)}=\frac{1}{2}\left[\gamma_{E}+\Gamma\left(0, \frac{z^{2} q^{2}}{4 k^{2}(s)}\right)+\ln \left(\frac{z^{2} q^{2}}{4 k^{2}(s)}\right)\right] .
$$

Furthermore, by changing variables to $y=z^{2} q^{2} /\left(4 k^{2}(s)\right)$ we find

$$
\int \frac{\mathrm{d} y}{(y+x)^{2}}\left(\gamma_{E}+\Gamma(0, y)-\ln y\right)=\frac{1}{x}\left[\gamma_{E}+\mathrm{e}^{x} \Gamma(0, x)+\ln x\right],
$$

where $x=z^{2} \mu^{2} /\left[4 k^{2}(s)\right]$. Finally, the second term of eq. (3.24) becomes

$$
\begin{aligned}
z \frac{\mathrm{d} I^{(1)}}{\mathrm{d} z}= & \frac{\alpha_{s} z P(z)}{\pi}\left(\frac{2 C_{R}}{N_{c}}-1\right) 2 \operatorname{Re} \int_{0}^{L} \mathrm{~d} s \\
& \times\left\{\frac{\hat{q}_{0}}{2 \mu^{2}}\left[\gamma_{E}+\mathrm{e}^{z^{2} \mu^{2} /\left[4 k^{2}(s)\right]} \Gamma\left(0, \frac{z^{2} \mu^{2}}{4 k^{2}(s)}\right)+\ln \frac{z^{2} \mu^{2}}{4 k^{2}(s)}\right]-\frac{z^{2} \hat{q}_{0}}{8 k^{2}(s)} \ln \frac{Q_{\text {sub }}^{2}}{\mu^{2}}\right\}
\end{aligned}
$$


where the first term appears due to the full elastic cross section, cf. eq. (3.26), while the second term is the subtraction of the harmonic oscillator term, cf. eq. (3.27). The first and third remaining terms can be found by simply substituting $z \rightarrow 1$ and $z \rightarrow 1-z$, respectively. Explicitly, the full NLO correction then takes the form

$$
\begin{aligned}
z \frac{\mathrm{d} I^{(1)}}{\mathrm{d} z}= & \frac{\alpha_{s} z P(z)}{\pi} 2 \operatorname{Re} \int_{0}^{L} \mathrm{~d} s \frac{\hat{q}_{0}}{2 \mu^{2}} \\
& \times\left\{F\left(\frac{\mu^{2}}{4 k^{2}(s)}\right)+\left(\frac{2 C_{R}}{N_{c}}-1\right) F\left(\frac{z^{2} \mu^{2}}{4 k^{2}(s)}\right)+F\left(\frac{(1-z)^{2} \mu^{2}}{4 k^{2}(s)}\right)\right\} \\
& -\frac{\alpha_{s} z P(z)}{\pi} 2 \operatorname{Re} \int_{0}^{L} \mathrm{~d} s \frac{\hat{q}_{\mathrm{eff}}}{4 k^{2}(s)} \ln \frac{Q_{\mathrm{sub}}^{2}}{\mu^{2}},
\end{aligned}
$$

where we introduced the shorthand

$$
F(x) \equiv \gamma_{E}+\mathrm{e}^{x} \Gamma(0, x)+\ln x .
$$

The full spectrum at NLO therefore becomes the sum of eqs. (3.18) and (3.31).

Let us investigate the two limits of the complex function $k^{2}(s)$, given in eq. (3.23). In the limit $\omega \gg \hat{q} L^{2}$, it follows that $\Omega \ll 1$, leading to $\tan (\Omega(L-s)) \approx 0$ and $\cot \Omega s \approx(\Omega s)^{-1}$. It follows that

$$
k^{2}(s) \simeq i \frac{\omega}{2 s},
$$

as in the vacuum. Then, expanding for small $x=\mu^{2} /\left[4 k^{2}(s)\right]$, we find

$$
\frac{1}{x}\left[\gamma_{E}+\mathrm{e}^{x} \Gamma(0, x)+\ln x\right] \approx 1-\gamma_{E}+\ln \frac{1}{x} .
$$

Hence, the spectrum in the high frequency regime $\omega \gg \hat{q} L^{2}$ becomes

$$
\omega \frac{\mathrm{d} I}{\mathrm{~d} \omega} \approx \bar{\alpha} \frac{\pi}{4} \frac{\hat{q} L^{2}}{\omega}
$$

which is a well-known limit of the GLV spectrum.

Turning to the small frequency regime, note that $k^{2}(s)$ also becomes vacuum-like since $Q_{\mathrm{sub}}^{2} \approx \mu^{2}$ for $\omega<\omega_{\mathrm{BH}}$ and therefore $\hat{q} \rightarrow 0$. We can now expand for large $x$, to find

$$
\omega \frac{\mathrm{d} I}{\mathrm{~d} \omega} \approx 2 \bar{\alpha} \frac{\hat{q}_{0} L}{\mu^{2}}\left(\ln \frac{\mu^{2} L}{2 \omega}-1+\gamma_{E}\right) .
$$

This is the characteristic behavior of the Bethe-Heitler regime, that is also contained in the GLV spectrum.

It is worth pointing out that in the regime $\omega \gg \omega_{\mathrm{BH}}$ that corresponds to $Q_{\mathrm{sub}}^{2} \gg \mu^{2}$ the dependence on the matching scale in of higher order and thus is weak. This is the leading logarithm dominated regime that was analyzed in detail up to NNLO in [26] in the improved opacity expansion scheme where the matching scale between the LPM and the UV regimes was precisely pinned down. However, at the $\mathrm{BH}$ scale, power corrections become sizable. Nevertheless, in order to provide an analytic formula at all scales for future 
phenomenological works one can interpolate between the two limits discussed above. Such an adjustment would be purely ad hoc at this stage. We leave the exact matching at the Bethe-Heitler frequency as an interesting future study, and focus here on a heuristically motivated interpolation between the three regimes, which we shall describe in more detail in the next section.

\section{$4 \quad$ Numerics}

Let us now return to the subtraction scale $Q_{\text {sub }}$ introduced earlier in the context the transport coefficient $\hat{q}=\hat{q}_{0} \ln a Q_{\text {sub }}^{2} / \mu^{2}$. As discussed in [23, 29], it is natural to define it in relation to the characteristic transverse momentum of the medium-induced emission process, i.e. $Q_{\text {sub }}^{2} \sim k_{\perp}^{2}$. For radiative processes, we have that $k_{\perp}^{2} \sim(\omega \hat{q})^{1 / 2} \sim$ $\left(\omega \hat{q}_{0} \ln \left(a \sqrt{\omega \hat{q}_{0}} / \mu^{2}\right)\right)^{1 / 2}$, since $\hat{q}$ itself is running with the subtraction scale. ${ }^{4}$ Crucially, to ensure the matching with the Bethe-Heitler spectrum at small gluon frequencies the latter logarithm should vanish for $Q^{2}<\mu^{2}$. This implies that $\hat{q} \rightarrow 0$ when $\sqrt{\omega \hat{q}}<\mu^{2}$ or $\omega<\omega_{B H} \equiv \mu^{2} \ell_{\mathrm{mfp}}$ (where we used that $\hat{q} \sim \mu^{2} / \ell_{\mathrm{mfp}}$ and we are left with a vacuum spectrum in this regime. To do so we use the following interpolating form

$$
Q_{\text {sub }}^{2}(\omega, \mu)=\sqrt{\omega \hat{q}_{0} \ln \left(\frac{a \sqrt{\omega \hat{q}_{0}}}{\mu^{2}}\right)} \mathrm{e}^{-\mu^{2} / \sqrt{\hat{q}_{0} \omega}}+\mu^{2} .
$$

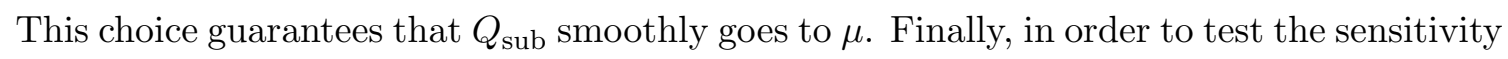
to the chosen matching, we will multiply the numerical factor $a$ by $1 / 2$ and 2 .

Let us first turn to the eikonal limit, where assume $x \ll 1$. We compare the results from our formula in the eikonal limit with the expectation from first order in opacity (for short, labelled "GLV") and the multiple soft scattering approximation (labelled "BDMPS") in figure 1. The spectrum interpolates well between the three physical regimes for inmedium QCD bremsstrahlung. For the choice of parameters here, corresponding to $\ell_{\mathrm{mfp}}<$ $L<\sqrt{E / \hat{q}}$, it clearly demonstrates that LPM interference effects are suppressing the spectrum over a large range of gluon energies. It is worth keeping in mind that we have only included the first order correction to the standard HO baseline, which leaves further room to improve on the matching by adding higher orders. The band around the "LO+NLO" curve corresponds to varying the matching parameter $a$ by a factor 2 up and down, which describes the inherent ambiguity in defining the LPM suppressed regime. Most importantly, our results reproduce the universal expectations at low- (corresponding to the Bethe-Heitler limit) and the high-frequency (corresponding to the "GLV" limit) and, all in all, the result over a wide frequency range is quite good.

The results for the improved spectrum with full $x$ dependence, calculated for a gluon jet with for three different energies $E=\{1000,250,62.5\} \mathrm{GeV}$, are given in figure 2. For the upper energy, the jet traverses a "thin" medium where $\sqrt{E / \hat{q}}>L$ while for the lower energy, the medium is "thick", i.e. $\sqrt{E / \hat{q}}<L$. This affects the range where the LPM is at

\footnotetext{
${ }^{4}$ Other choices are indeed possible, e.g. fixing $Q_{\text {sub }}^{2} \sim \hat{q} L \sim \hat{q}_{0} L \ln \left(\hat{q}_{0} L / \tilde{\mu}^{2}\right)$, which is the expected behavior at high energies $\omega>\hat{q} L^{2}$. Alternatively, we could also demand that the scale saturates, i.e. $Q_{\text {sub }}^{2} \leq \hat{q} L$
} 


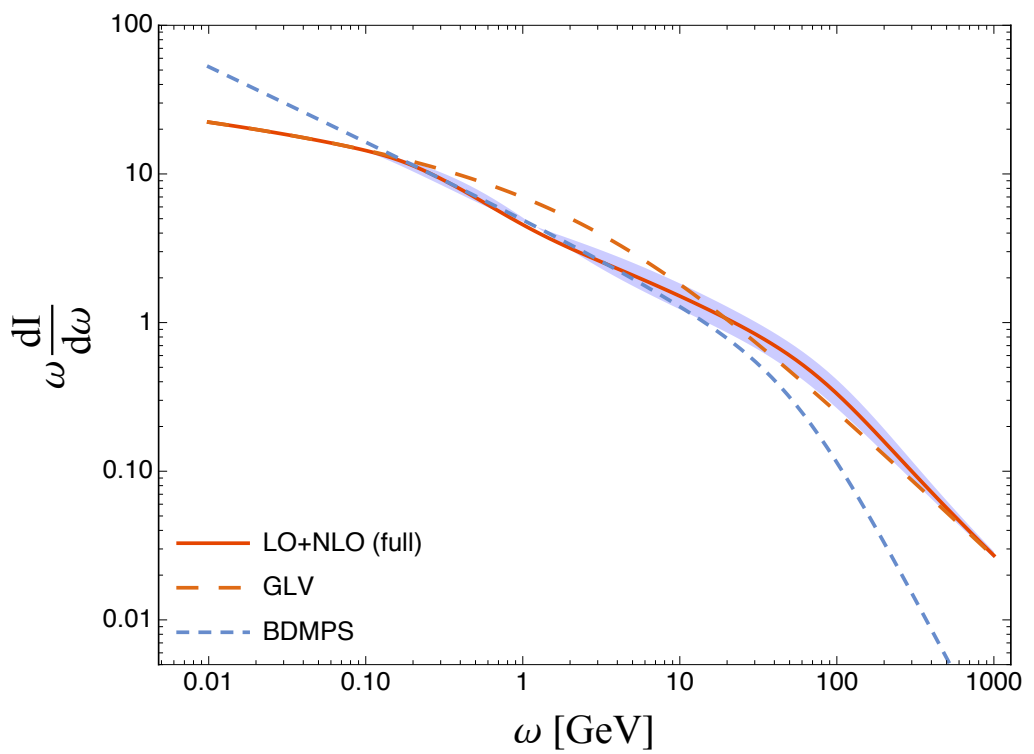

Figure 1. The eikonal spectrum (assuming $x \ll 1$ ) up to next-to-leading order, compared to GLV and BDMPS for a jet with energy $E=1000 \mathrm{GeV}$. Medium parameters are $\hat{q}=1.5 \mathrm{GeV}^{2} / \mathrm{fm}$, $\mu=1 \mathrm{GeV}$ and $L=4 \mathrm{fm}$.

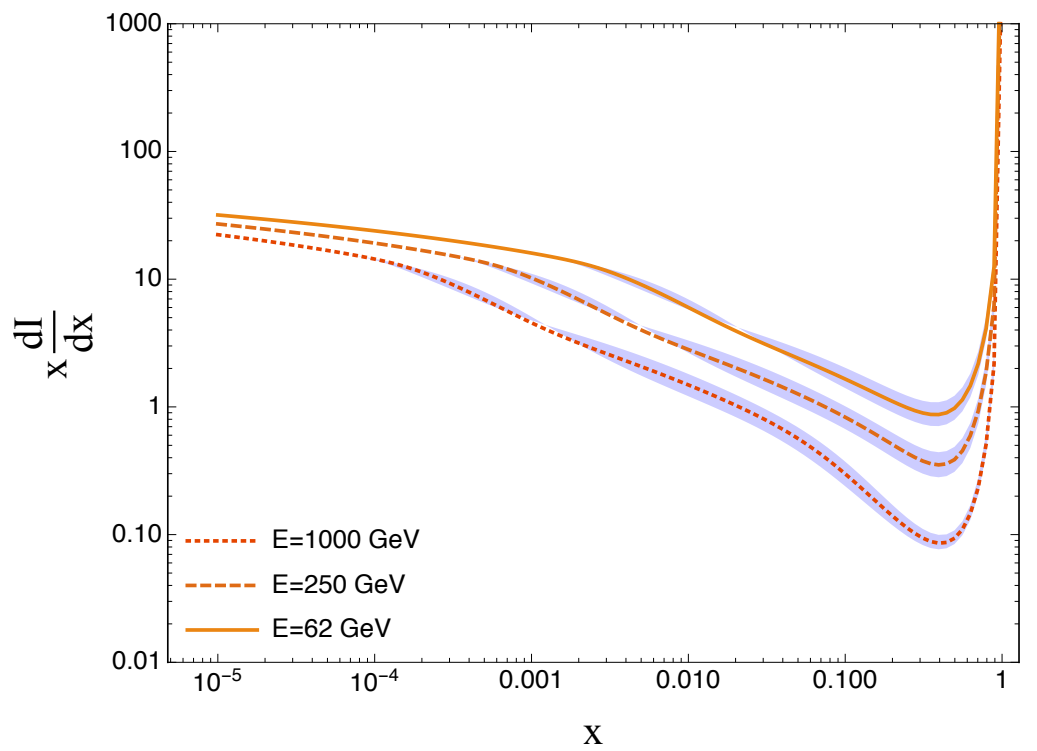

Figure 2. The matched spectrum up to next-to-leading order, compared to GLV and BDMPS for a jet with energy $E=250 \mathrm{GeV}$ (top) and $E=1000 \mathrm{GeV}$ (bottom). Medium parameters are $\hat{q}=1.5 \mathrm{GeV}^{2} / \mathrm{fm}, \mu=1 \mathrm{GeV}$ and $L=4 \mathrm{fm}$.

play. The turning over of the curves at small- $x$ correspond to the $\mathrm{BH}$ regime which takes place at a fixed gluon energy $\omega_{\mathrm{BH}}$, not a fixed $x$ for different jet energies.

We have also computed numerically the rate of emissions, defined as $\mathrm{d} I /(\mathrm{d} \omega \mathrm{d} t)$, in figure 3. As expected, at small times the rate grows linearly with time like the GLV spectrum, i.e. $\propto t$. At later times the rate satures in the LPM regime. We observe the 


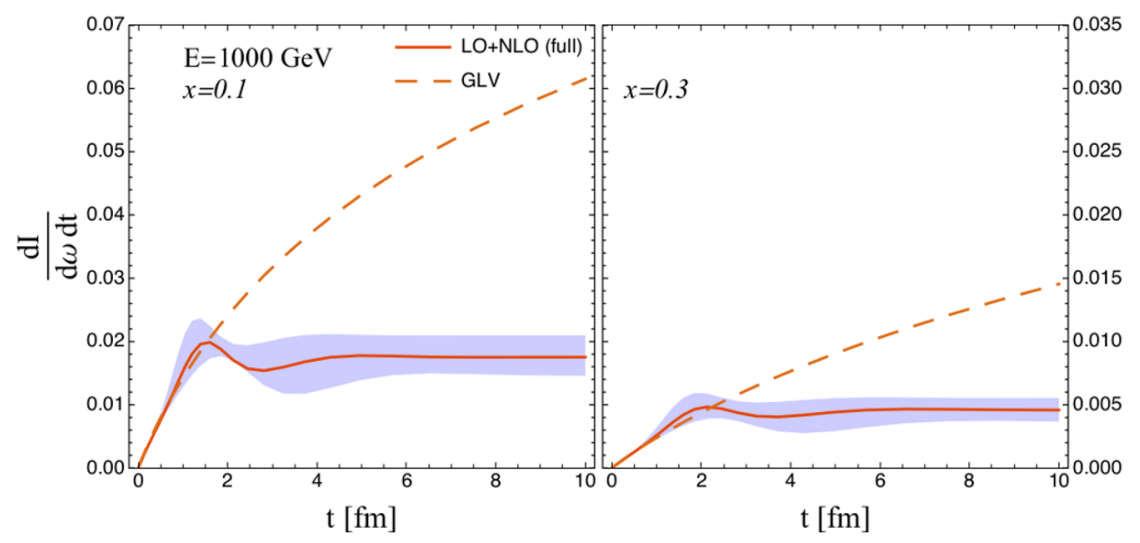

Figure 3. The rate of medium-induced emissions for a gluon jet with energy $E=1 \mathrm{TeV}$. Medium parameters are $\hat{q}=1.5 \mathrm{GeV}^{2} / \mathrm{fm}$ and $\mu=1 \mathrm{GeV}$.

uncertainty related to the implementation of the LPM regime as a band that predominantly appears whenever the rate saturates. For the same reason, we have therefore chosen not to plot the expectation from the "bare" BDMPS spectrum since it would differ from our curve by a constant offset related to choice of scale in $\hat{q}$.

Finally, in order to compare our compact, analytical formula with the full, all-order in opacity solution of the spectrum, solved numerical in [19], we plot in figure 4 the rate for two choices of medium parameters and jet kinematics. Since our result only includes the first corrections from hard scattering, the agreement is reasonable $(\lesssim 30 \%)$. Note that some of the discrepancy may be attributed to the different choices of the potential and the lack of thermal masses in our approach.

\section{Conclusions}

In this work, we revisit the calculation of medium-induced parton splitting and present a new analytic approach that allows for the first time to account for the various known limits. Our method, dubbed "Improved Opacity expansion" resums multiple soft scatterings to all orders while treating single hard scattering as a perturbation. To do so, following [23], we have suggested a shift of the expansion of the in-medium propagators around the so-called "harmonic oscillator" solution which takes into account diffusive momentum broadening. Perturbations around this solution correspond to hard, transverse kicks that reveal the quasi-particle structure of the underlying medium. In ref. [23], the radiative spectrum was calculated in the leading logarithmic approximation and therefore is applicable so long as $x_{\perp} \ll \mu^{-1}$, which translates into $\omega \gg \omega_{\mathrm{BH}}$. In the present work we go beyond by accounted for the full imaginary potential order by order.

We have demonstrated the validity of the framework by computing the in-medium radiative spectrum. The NLO term, with a suitable choice of subtraction scale, allows to properly link the LPM regime, appropriate for dense media, to the regimes where singlescattering in the medium dominates, including the Bethe-Heitler regime at low frequencies, where formation time of the radiation probes the scale of the medium mean-free-path, and 

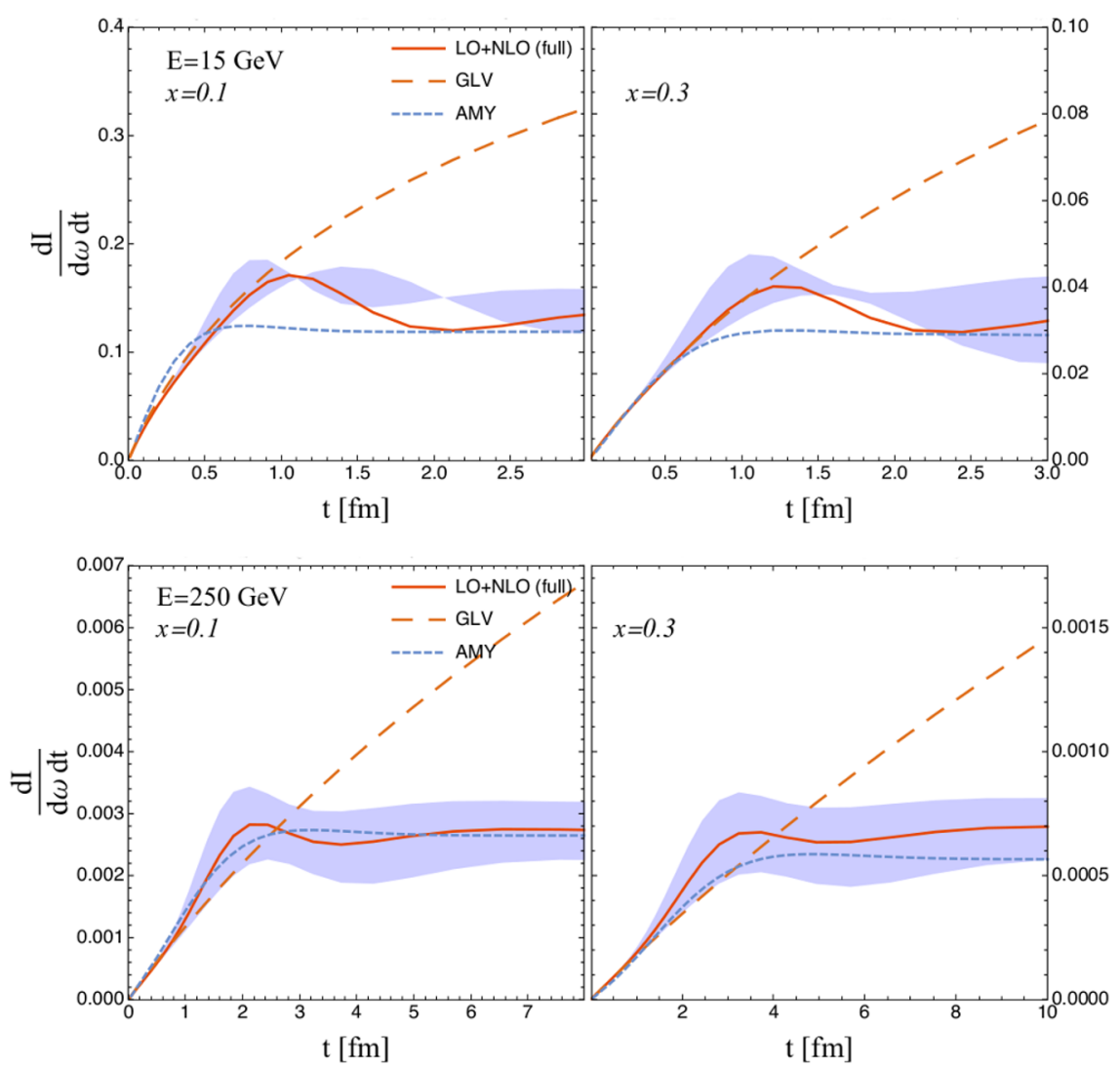

Figure 4. The rate of medium-induced emissions for a jet with energy $E=15 \mathrm{GeV}$ (upper panel) and $E=250 \mathrm{GeV}$ (lower panel). Medium parameters are $\hat{q}=1.63 \mathrm{GeV}^{2} / \mathrm{fm}$ and $\mu=0.9 \mathrm{GeV}$, corresponding to $g=1.94$ and $T=0.4 \mathrm{GeV}$. The dashed (blue) lines labelled "AMY" correspond to the numerical evaluation presented in [19].

the GLV regime at high frequencies, where the formation time of the bremsstrahlung exceeds the length of the medium. This demonstrates that the approach, albeit formally suitable for large and dense media, where diffusive broadening dominate at small angles. The compactness of our main result and the good agreement with the results from an all-order resummation [19], which is implemented in the MARTINI event generator [33, 34], makes it amenable for implementation in a fast Monte-Carlo event generator for jet quenching.

\section{Acknowledgments}

The authors thank Sangyong Jeon, Chanwook Park, Simon Caron-Huot and Charles Gale for discussions and for providing numerical results for the code used in [29]. KT is supported by a Starting Grant from Trond Mohn Foundation (BFS2018REK01) and the University of Bergen. YMT is supported by the U.S. Department of Energy, Office of Science, Office of Nuclear Physics, under contract No. DE- SC0012704, and in part by Laboratory Directed Research and Development (LDRD) funds from Brookhaven Science Associates. 
Open Access. This article is distributed under the terms of the Creative Commons Attribution License (CC-BY 4.0), which permits any use, distribution and reproduction in any medium, provided the original author(s) and source are credited.

\section{References}

[1] S. Peigne and A.V. Smilga, Energy losses in a hot plasma revisited, Phys. Usp. 52 (2009) 659 [arXiv:0810.5702].

[2] Y. Mehtar-Tani, J.G. Milhano and K. Tywoniuk, Jet physics in heavy-ion collisions, Int. J. Mod. Phys. A 28 (2013) 1340013 [arXiv:1302.2579] [INSPIRE].

[3] J.-P. Blaizot and Y. Mehtar-Tani, Jet Structure in Heavy Ion Collisions, Int. J. Mod. Phys. E 24 (2015) 1530012 [arXiv: 1503.05958] [INSPIRE].

[4] R. Baier, Y.L. Dokshitzer, A.H. Mueller, S. Peigne and D. Schiff, Radiative energy loss of high-energy quarks and gluons in a finite volume quark-gluon plasma, Nucl. Phys. B 483 (1997) 291 [hep-ph/9607355] [INSPIRE].

[5] R. Baier, Y.L. Dokshitzer, A.H. Mueller, S. Peigne and D. Schiff, Radiative energy loss and $p_{T}$ broadening of high-energy partons in nuclei, Nucl. Phys. B 484 (1997) 265 [hep-ph/9608322] [INSPIRE].

[6] B.G. Zakharov, Fully quantum treatment of the Landau-Pomeranchuk-Migdal effect in QED and QCD, JETP Lett. 63 (1996) 952 [hep-ph/9607440] [INSPIRE].

[7] B.G. Zakharov, Radiative energy loss of high-energy quarks in finite size nuclear matter and quark-gluon plasma, JETP Lett. 65 (1997) 615 [hep-ph/9704255] [INSPIRE].

[8] P.B. Arnold, G.D. Moore and L.G. Yaffe, Photon and gluon emission in relativistic plasmas, JHEP 06 (2002) 030 [hep-ph/0204343] [INSPIRE].

[9] U.A. Wiedemann, Gluon radiation off hard quarks in a nuclear environment: Opacity expansion, Nucl. Phys. B 588 (2000) 303 [hep-ph/0005129] [INSPIRE].

[10] M. Gyulassy, P. Levai and I. Vitev, Reaction operator approach to nonAbelian energy loss, Nucl. Phys. B 594 (2001) 371 [nucl-th/0006010] [INSPIRE].

[11] G. Ovanesyan and I. Vitev, Medium-induced parton splitting kernels from Soft Collinear Effective Theory with Glauber gluons, Phys. Lett. B 706 (2012) 371 [arXiv:1109.5619] [INSPIRE].

[12] M.D. Sievert and I. Vitev, Quark branching in QCD matter to any order in opacity beyond the soft gluon emission limit, Phys. Rev. D 98 (2018) 094010 [arXiv: 1807.03799] [INSPIRE].

[13] M.D. Sievert, I. Vitev and B. Yoon, A complete set of in-medium splitting functions to any order in opacity, Phys. Lett. B $\mathbf{7 9 5}$ (2019) 502 [arXiv:1903.06170] [INSPIRE].

[14] X.-N. Wang and X.-f. Guo, Multiple parton scattering in nuclei: Parton energy loss, Nucl. Phys. A 696 (2001) 788 [hep-ph/0102230] [INSPIRE].

[15] A. Majumder, Hard collinear gluon radiation and multiple scattering in a medium, Phys. Rev. D 85 (2012) 014023 [arXiv:0912.2987] [INSPIRE].

[16] P.B. Arnold and C. Dogan, QCD Splitting/Joining Functions at Finite Temperature in the Deep LPM Regime, Phys. Rev. D 78 (2008) 065008 [arXiv:0804.3359] [inSPIRE]. 
[17] B.G. Zakharov, On the energy loss of high-energy quarks in a finite size quark-gluon plasma, JETP Lett. 73 (2001) 49 [hep-ph/0012360] [INSPIRE].

[18] P.B. Arnold, High-energy gluon bremsstrahlung in a finite medium: harmonic oscillator versus single scattering approximation, Phys. Rev. D 80 (2009) 025004 [arXiv:0903.1081] [INSPIRE].

[19] S. Caron-Huot and C. Gale, Finite-size effects on the radiative energy loss of a fast parton in hot and dense strongly interacting matter, Phys. Rev. C 82 (2010) 064902 [arXiv: 1006.2379$]$ [INSPIRE].

[20] X. Feal and R. Vazquez, Intensity of gluon bremsstrahlung in a finite plasma, Phys. Rev. D 98 (2018) 074029 [arXiv: 1811.01591] [INSPIRE].

[21] W. Ke, Y. Xu and S.A. Bass, Modified Boltzmann approach for modeling the splitting vertices induced by the hot QCD medium in the deep Landau-Pomeranchuk-Migdal region, Phys. Rev. C 100 (2019) 064911 [arXiv:1810.08177] [INSPIRE].

[22] C. Andres, L. Apolinário and F. Dominguez, Medium-induced gluon radiation with full resummation of multiple scatterings for realistic parton-medium interactions, arXiv: 2002.01517 [INSPIRE].

[23] Y. Mehtar-Tani, Gluon bremsstrahlung in finite media beyond multiple soft scattering approximation, JHEP 07 (2019) 057 [arXiv: 1903.00506] [INSPIRE].

[24] G. Moliere, Theory of the scattering of fast charged particles. 2. Repeated and multiple scattering, Z. Naturforsch. A 3 (1948) 78.

[25] E. Iancu, K. Itakura and D.N. Triantafyllopoulos, Cronin effect and high p-perpendicular suppression in the nuclear gluon distribution at small x, Nucl. Phys. A $\mathbf{7 4 2}$ (2004) 182 [hep-ph/0403103] [INSPIRE].

[26] J. Barata and Y. Mehtar-Tani, Improved opacity expansion at NNLO for medium induced gluon radiation, arXiv: 2004.02323 [INSPIRE].

[27] P. Aurenche, F. Gelis and H. Zaraket, A simple sum rule for the thermal gluon spectral function and applications, JHEP 05 (2002) 043 [hep-ph/0204146] [INSPIRE].

[28] P.B. Arnold, G.D. Moore and L.G. Yaffe, Transport coefficients in high temperature gauge theories. 2. Beyond leading log, JHEP 05 (2003) 051 [hep-ph/0302165] [INSPIRE].

[29] S. Caron-Huot, O(g) plasma effects in jet quenching, Phys. Rev. D 79 (2009) 065039 [arXiv: 0811.1603] [INSPIRE].

[30] X.-N. Wang and M. Gyulassy, Gluon shadowing and jet quenching in $A+A$ collisions at $\sqrt{s}=200 \mathrm{GeV}$, Phys. Rev. Lett. 68 (1992) 1480 [inSPIRE].

[31] M. Abramowitz and I. Stegun eds., Handbook of Mathematical Functions, Dover Publications, New York U.S.A. (1965).

[32] P.B. Arnold, Simple Formula for High-Energy Gluon Bremsstrahlung in a Finite, Expanding Medium, Phys. Rev. D 79 (2009) 065025 [arXiv:0808.2767] [InSPIRE].

[33] B. Schenke, C. Gale and S. Jeon, MARTINI: An Event generator for relativistic heavy-ion collisions, Phys. Rev. C 80 (2009) 054913 [arXiv: 0909.2037] [INSPIRE].

[34] C. Park, S. Jeon and C. Gale, Jet modification with medium recoil in quark-gluon plasma, Nucl. Phys. A 982 (2019) 643 [arXiv:1807.06550] [INSPIRE]. 